\title{
CIERRE DE DUCTUS ARTERIOSO PERSISTENTE EN NEONATOS PRETÉRMINO \\ USO DE DICLOFENACO SÓdICO ENDOVENOSO, HOSPITAL DE SAN JOSÉ, BOGOTÁ DC
}

Sandra Patricia Albornoz MD*, José María Campo MD*, Rubén Darío García MD*, Yosiris del Carmen Florián MD*, Héctor Romero MD**

\section{Resumen}

El ductus arterioso persistente (DAP) aumenta el riesgo de hemorragia intraventricular, enterocolitis necrosante, displasia broncopulmonar y muerte. La indometacina se ha considerado el medicamento de elección para su cierre farmacológico, pero la imposibilidad de importarla al país motivó el uso de diclofenaco sódico como alternativa. Objetivo: uso de diclofenaco endovenoso para el cierre del DAP en neonatos pretérmino en el Hospital de San de José de Bogotá, de febrero de 2007 a junio de 2010. Diseño y metodología: estudio descriptivo retrospectivo de 38 recién nacidos pretérmino de 0 a 20 días de edad posnatal con diagnóstico de DAP, repercusión hemodinámica y/o diámetro mayor o igual a $2 \mathrm{~mm}$ y que recibieron diclofenaco para el cierre farmacológico. Resultados: con un solo ciclo del medicamento hubo cierre en el $\mathbf{8 1 , 5 \%}$. El $\mathbf{6 5 , 8 \%}$ requirieron menos de siete días de soporte ventilatorio. Las principales complicaciones fueron renales y neurológicas, con escasas reacciones adversas. Conclusiones: el diclofenaco mostró un buen porcentaje de cierre de DAP con bajos efectos adversos, por lo cual podría considerarse como alternativa para el manejo de esta patología cuando la terapia estándar no está disponible, pero consideramos necesarios nuevos estudios comparativos para obtener conclusiones relevantes.

Palabras clave: ductus arterioso persistente, diclofenaco, neonatos.

Abreviaturas: DAP, ductus arterioso persistente; RN, recién nacido.

\section{CLOSURE OF PERSISTENT ARTERIAL DUCT IN PRETERM NEWBORNS. USE OF IV DICLOFENAC SODIUM -}

\section{hOSPITAL DE SAN JOSÉ BOGOTÁ DC.}

\begin{abstract}
Persistent arterial duct (PAD) increases the risk of intraventricular hemorrhage, necrotizing enteropathy, bronchial/ pulmonary dysplasia and death. Indomethacin is considered the preferred drug for pharmacological closure of PAD. Diclofenac sodium is used as an alternative due to import-related restrictions for indomethacin in our country. Objective: use of IV diclofenac for PAD closure in preterm newborns at Hospital de San de José, Bogotá, from February 2007 to June 2010. Design and Methodology: this is a descriptive retrospective study conducted in 38 preterm newborns 0 to 20 days after birth who have been diagnosed with PAD, hemodynamic repercussion, and/or major diameter greater than $2 \mathrm{~mm}$, who received diclofenac for pharmacologic closure. Results: closure was achieved with only one cycle in $\mathbf{8 1 . 5 \%}$. 65.8\% required less than seven days of ventilatory support. Major complications were renal and neurological, with few adverse reactions. Conclusions: diclofenac showed a good rate of PAD closure with few adverse effects, thus it can be considered an alternative to handle this condition when standard therapy is not available. Nonetheless, we consider new comparative studies are necessary in order to obtain relevant conclusions.
\end{abstract}

Key words: persistent arterial ductus, diclofenac, newborns.

Fecha recibido: marzo 31 de 2011 - Fecha aceptado: junio 10 de 2011

* Residente III de Pediatría, Fundación Universitaria de Ciencias de la Salud, Hospital de San José, Bogotá DC. Colombia.
** Profesor Asistente, Fundación Universitaria de Ciencias de la Salud. Jefe del Servicio de Pediatría, Clínica Federmán. Bogotá DC, Colombia. 


\section{Introducción}

El DAP representa la permanencia del conducto que conecta el origen de la arteria pulmonar con la aorta, permitiendo el paso de sangre de la primera a la segunda, de tal forma que solo el 10 a $15 \%$ del gasto cardíaco combinado pasa por los pulmones durante la vida fetal. Después del nacimiento se genera una caída en los niveles de prostaglandinas (E1, I2, E2) y ocurre el cierre espontáneo del DAP en los primeros tres días de vida. ${ }^{1,2,3}$ A nivel del mar el cierre funcional se presenta entre 8 y 48 horas $(90 \%)$ y anatómico a los 21 días, pero a mayor altura este cierre es más tardío, por ejemplo en Bogotá se estima que hay algunos con mínima permeación a los dos meses de vida y cerrados en su totalidad inclusive hasta los tres meses. El mecanismo implicado en este cierre se debe a que en el momento de la primera respiración del RN, por efecto vasodilatador del oxígeno en los vasos pulmonares, hay disminución de la resistencia pulmonar con un aumento del flujo generando un incremento del retorno venoso pulmonar a la aurícula izquierda, a su vez, la resistencia vascular sistémica se eleva al cortar el flujo sanguíneo placentario, con lo cual se establece un gradiente de presión a favor de la aorta. Todo ello permite un cortocircuito de izquierda a derecha a través del ductus, contrario a lo que ocurría en la vida fetal, el cual era de derecha a izquierda. ${ }^{1,2,3}$

Debido a todos estos cambios, la sangre que pasa por el ductus proveniente de la aorta contiene una saturación de oxígeno elevada (mayor de $90 \%$ ), generando un aumento brusco de la concentración de oxígeno a nivel ductal en cerca de 20 a 40 torr, produciendo el cierre funcional por contracción de la musculatura lisa y alteración de la nutrición de la pared del ductus, llevando a una degeneración isquémica con necrosis de la pared y cambios citolíticos, donde después proliferan los fibroblastos que llevan a que se convierta en el ligamento arterioso. ${ }^{4,5,6}$ La persistencia tras el nacimiento se debe a que los prematuros poseen un bajo tono muscular, al igual que un menor número de fibras musculares ductales en comparación con los $\mathrm{RN}$ a término. Desde el punto de vista microscópico, el DAP tiene dos capas musculares con orientación en espiral y su constitución es diferente de la aorta y la pulmonar. ${ }^{6}$
Las consecuencias clínicas del DAP se relacionan en forma directa con el grado de derivación de izquierda a derecha a través del conducto. Pese a la capacidad del ventrículo izquierdo en los $\mathrm{RN}$ prematuros de aumentar su gasto frente a una derivación de izquierda a derecha, la distribución del flujo sanguíneo a los órganos vitales se ve alterado por la disminución de la presión diastólica y a la vasoconstricción localizada.? Una derivación importante de izquierda a derecha a través del conducto puede aumentar el riesgo de hemorragia intraventricular, enterocolitis necrosante, displasia broncopulmonar y muerte. ${ }^{3,7}$

La incidencia del DAP varía en función de la edad gestacional y del peso al nacimiento y aumenta a medida que sean menores estos factores; en prematuros de muy bajo peso con síndrome de dificultad respiratoria se aproxima al $40 \%$ en el tercer día de vida. Factores como el uso de ventilación mecánica aumenta su incidencia, ${ }^{8,9} \sin$ olvidar que es necesario mantener el DAP en algunos tipos de cardiopatías dependientes del mismo para generar un flujo sistémico adecuado. En el prematuro el cuadro clínico varía cuando se presenta en forma aislada o asociado al síndrome de dificultad respiratoria; en el primer caso el diagnóstico debe sospecharse ante el deterioro del estado general, con aparición de polipnea, pulso saltón, hiperdinamia cardíaca, hepatomegalia congestiva y auscultación de soplo en el tercio superior o medio del borde esternal izquierdo, el cual por lo regular es sistólico con o sin refuerzo telesistólico. Con menor frecuencia se ausculta un pequeño soplo sistólico en región interescapular izquierda, ante lo cual debe sospecharse DAP y solicitar ecocardiograma con radiografía de tórax y electrocardiograma. ${ }^{6,89}$ En el segundo caso, ante el compromiso pulmonar como la enfermedad de membrana hialina, el diagnóstico puede tornarse difícil porque el cuadro pulmonar absorbe toda la atención; sin embargo, esta asociación debe tenerse siempre en mente. Se considera el ecocardiograma como prueba de oro para el diagnóstico de DAP en el RN, por ser un estudio no invasivo, de fácil acceso, económico sensible y específico.

La reducción de la síntesis de prostaglandinas mediante los inhibidores no selectivos de ciclooxigenasas 1 y 2 ha sido efectiva para el cierre no quirúrgico del 
DAP. ${ }^{7,10}$ Desde 1976 la indometacina se ha considerado de elección para el cierre farmacológico, pero se han descrito efectos adversos en especial a nivel renal, digestivo y del flujo sanguíneo cerebral. ${ }^{4}$ Por ello, se han estudiado otros inhibidores de la cicloxigenasa, sobre todo durante la última década. El ibuprofeno intravenoso ha demostrado ser igual de eficaz para el cierre del DAP en el RN pretérmino y presenta menor repercusión a nivel renal, habiéndose aprobado su uso en esta patología. ${ }^{1,11} \mathrm{La}$ indometacina intravenosa es el tratamiento estándar para el cierre del DAP en RN prematuros y se ha utilizado desde $1976^{1,4,7,9,11}$ con una eficacia informada del 66 al $80 \%$. $^{6,12}$ Esta terapia debe realizarse solo cuando exista repercusión hemodinámica y en estos casos no se debe retrasar su uso, pues actúa mejor cuanto más temprano se inicie y mayor inmadurez presente el RN. ${ }^{10,13}$ Por lo regular no actúa después de los veinte días de vida y no debe emplearse en los niños a término. ${ }^{7,10}$

El uso de indometacina puede producir efectos secundarios como disminución del flujo sanguíneo cerebral y del aporte de oxígeno al sistema nervioso central, oliguria o insuficiencia renal transitoria, enterocolitis necrosante, perforación intestinal aislada o hemorragia gastrointestinal..$^{1,7,11-13}$ En general los inhibidores de la ciclooxigenasa están contraindicados en sepsis, hemorragia, enterocolitis y alteraciones de la función renal.

Los $\mathrm{RN}$ con cardiopatías congénitas complejas, asfixia severa, alteraciones genéticos y malformaciones mayores no son los mejores candidatos y cada caso debe ser evaluado en forma particular., ${ }^{70,14}$ Se debe realizar recuento de plaquetas y pruebas de función renal antes de su uso y se puede iniciar con valores de creatinina máximo en $1.5 \mathrm{mg} / \mathrm{dl}$. Debido a la contraindicación de sangrado, se debe realizar antes una ecografía cerebral para descartar hemorragia cerebral. Si hay trombocitopenia, salvo en casos de sepsis, se debe transfundir con hemoderivados y luego se inicia la dosis respectiva. ${ }^{15}$ En menores de mil gramos ante cualquier signo de repercusión hemodinámica deben iniciarse de inmediato los inhibidores de la ciclooxigenasa. ${ }^{8,12,13,16}$ Se recomienda el siguiente esquema: inferiores a $1.000 \mathrm{~g}$ : tres dosis de 0,1 a $0,15 \mathrm{mg} / \mathrm{k}$ cada doce horas; de 1.000 a $1.500 \mathrm{~g}: 0,15$ a $0,2 \mathrm{mg} / \mathrm{k}$ cada ocho horas; de 1.500 a $2.000 \mathrm{~g}$ : 0,2 a $0,25 \mathrm{mg} / \mathrm{k}$ cada ocho horas; y superiores a $2.000 \mathrm{~g}: 0.25 \mathrm{mg} / \mathrm{k}$ cada ocho horas. ${ }^{7,12,16}$

Estas complicaciones han hecho que se investiguen otras estrategias terapéuticas más seguras para el cierre del DAP ${ }^{10,14,16}$ como el ibuprofeno. ${ }^{7,10,14}$ En nuestro medio e institución debido a la dificultad para la consecución, a partir de marzo de 2008 se ha venido usando el diclofenaco sódico endovenoso para el cierre del DAP en estos pacientes. Por ello, se decidió realizar un estudio retrospectivo observacional de tipo descriptivo con el objetivo de informar sobre la experiencia obtenida con esta nueva opción del grupo farmacológico similar. Concluimos que dada la efectividad y el mayor beneficio, se debe intentar el cierre farmacológico antes que la posibilidad quirúrgica en todos los RN con DAP, siempre y cuando no exista una contraindicación.

\section{Materiales y métodos}

Se realizó un estudio retrospectivo observacional de tipo descriptivo que abarcó el período comprendido entre el primero de febrero de 2007 al treinta de junio de 2010. Se evaluaron los casos de prematuros con DAP. Se solicitaron al departamento de registros médicos 158 historias clínicas obtenidas de los archivos de ecocardiogramas (diagnosticados con DAP sin tener en cuenta criterios de inclusión al estudio) hechos en la institución para el período en cuestión, de estas 38 cumplieron los criterios de inclusión, los cuales fueron: $\mathrm{RN}$ pretérmino menor o igual a 37 semanas, de cero a veinte días de edad posnatal, hospitalizado en la unidad de RN del Hospital de San José, con diagnóstico de DAP confirmado por ecocardiograma, llevado a cierre farmacológico con diclofenaco, repercusión hemodinámica (precordio hiperdinámico, hipertensión pulmonar, signos de falla cardíaca) con criterio clínico definido por el médico pediatra o neonatólogo de la unidad de RN y/o diámetro del DAP mayor o igual a 2 $\mathrm{mm}$, cuyo diagnóstico ecocardiográfico hubiera sido realizado por el cardiólogo pediatra. Los criterios de exclusión fueron malformación cardíaca congénita dependiente del ductus, $\mathrm{RN}$ con criterios para cierre quirúrgico primario (malformación cardíaca mayor, 
inestabilidad hemodinámica) e insuficiencia renal. La información incluyó las características sociodemográficas de la población como edad gestacional y posnatal, género, peso, inicio del tratamiento y tiempo de diagnóstico ecocardiográfico según la edad gestacional y posnatal (Tabla 1).

Las características en relación con el tratamiento con diclofenaco (inicio en horas de la primera dosis, reapertura, cierre quirúrgico, porcentaje de cierre y número de ciclos) se muestran en la Tabla 2. Las

\begin{tabular}{|c|c|c|}
\hline & Frecuencia & Total (\%) \\
\hline \multicolumn{3}{|l|}{ Género } \\
\hline masculino & 19 & $(50)$ \\
\hline \multicolumn{3}{|l|}{ Edad gestacional } \\
\hline pretérmino $30-37$ sem & 19 & (50) \\
\hline pretérmino $<30$ sem & 19 & $(50)$ \\
\hline \multicolumn{3}{|l|}{ Peso } \\
\hline$<1.000 \mathrm{~g}$ & 9 & $(23.6)$ \\
\hline $1.000-1.500 \mathrm{~g}$ & 13 & (34.2) \\
\hline $1.500-2.000 \mathrm{~g}$ & 6 & (I5.7) \\
\hline$>2.000 \mathrm{~g}$ & 10 & $(26.3)$ \\
\hline
\end{tabular}

\begin{tabular}{|lcc|}
\hline \multicolumn{4}{|c|}{ Tabla 2. Tratamiento con diclofenaco } \\
\hline \multicolumn{4}{|c|}{$(\%)$} \\
\hline Inicio del ciclo de diclofenaco por horas de vida \\
$<24$ horas & 7 & $(18.4)$ \\
$24-72$ horas & 13 & $(34.3)$ \\
$>72$ & 18 & $(47.3)$ \\
Número de ciclos de diclofenaco usados & \\
Uno & 37 & $(97.4)$ \\
Dos & 1 & $(2.6)$ \\
Cierre DAP confirmado por ecocardiograma & \\
Si & 31 & $(81.5)$ \\
No & 3 & $(7.8)$ \\
Sin datos* & 4 & $(10.7)$ \\
Reapertura de DAP & & \\
Sí & 3 & $(7.8)$ \\
No & 31 & $(81.5)$ \\
Indeterminado* & 4 & $(10.7)$ \\
Remitido para cierre quirúrgico & & \\
No & 35 & $(92.2)$ \\
\hline
\end{tabular}

* Neonatos que fallecieron antes de realizar el ecocardiograma de control postratamiento farmacológico.

\begin{tabular}{|lcc|}
\hline \multicolumn{2}{|c|}{ Tabla 3. Reacciones y efectos adversos } \\
\hline & $\mathbf{n}$ & $(\%)$ \\
Reacciones adversas & 1 & $(2,6)$ \\
eritema & 0 & $(0)$ \\
exantema & 2 & $(5.2)$ \\
sangrado & & \\
Efectos adversos & 6 & $(15.7)$ \\
hemorragia intraventricular & 3 & $(7.8)$ \\
oliguria & 1 & $(3.8)$ \\
perforación gástrica* & 6 & $(15.7)$ \\
falla renal & 0 & $(0)$ \\
enterocolitis necrosante & & \\
\hline
\end{tabular}

* Solo un caso de perforación gástrica en pretérmino de muy bajo peso y factores de riesgo con empleo de corticoide y sepsis.

\begin{tabular}{|lcc|}
\hline \multicolumn{3}{|c|}{ Tabla 4. Resultados } \\
\hline \\
\hline Fallecidos & ( $\%)$ \\
Si & 8 & $(21)$ \\
Tiempo de ventilación mecánica & & \\
$<4$ días & 16 & $(42.2)$ \\
4 a 7 días & 9 & $(23.6)$ \\
Tiempo de suplencia de oxígeno & & \\
$<8$ días & 5 & $(13.1)$ \\
8 a 28 & 19 & $(50)$ \\
$>28$ dias & 14 & $(36.9)$ \\
Tiempo de estancia hospitalaria & & \\
$<8$ días & 4 & $(10.6)$ \\
8 a 28 & 17 & $(44.7)$ \\
$>28$ días & 17 & $(44.7)$ \\
\hline
\end{tabular}

reacciones adversas y complicaciones de la terapéutica se muestran en la Tabla 3. Los resultados en relación con el desenlace se muestran en la Tabla 4.

\section{Discusión}

El objetivo primario de este estudio fue describir la experiencia obtenida tras el uso del diclofenaco endovenoso para el cierre del DAP en RN prematuros. El medicamento logró mostrar alta capacidad de cierre del DAP $(81,5 \%)$, pues según la literatura el porcentaje de efectividad con indometacina está entre 50 y $60 \%{ }^{6,9}$ 
En la mayoría de los casos se requirió un solo ciclo de tratamiento y en un bajo porcentaje ante la reapertura del ductus, por lo cual constituye una buena opción para esta patología sin convertirlo en un remplazo de las indicaciones farmacológicas vigentes en la actualidad. $7,10,12,14,16$

En la población estudiada no se evidenció predominio de género, la distribución según la edad gestacional fue igual en los pretérminos menores de 30 semanas y el grupo de 30 a 37 semanas. Hubo mayor presentación de casos en el grupo de pretérmino con peso inferior a $1.500 \mathrm{~g}$, haciendo evidente una relación entre menor peso al nacer y mayor incidencia del DAP como se recalca en la literatura médica. ${ }^{7,8,12,14}$ No se puede deducir en nuestro estudio que el inicio de la terapia farmacológica antes de 72 horas del diagnóstico ecocardiográfico, tenga mejores resultados en la variable cierre del ductus. ${ }^{7,9,13,16}$

En humanos se conoce el efecto del uso con diclofenaco sódico en estadios prenatales y sus consecuencias en el cierre del DAP; sin embargo, cabe recalcar su contraindicación en estas etapas de la vida por sus posibles consecuencias mortales, ${ }^{17-22}$ no hay evidencia suficiente del uso de diclofenaco en RN para el cierre del DAP. Entre las complicaciones encontradas en el estudio se evidenció mayor compromiso a nivel neurológico (hemorragia intraventricular) y renal (oliguria, falla renal), con seis casos $(15,7 \%)$ del primero comparado con 17 a 57,5\% reportado en los estudios con indometacina ${ }^{6,9}$, tres casos $(7,8 \%)$ con oliguria en relación con el $19 \%$ informado con indometacina $^{6,9}$; hubo seis casos $(15,7 \%)$ de falla renal. Estos resultados podrían explicarse por pertenecer al grupo farmacológico de inhibidores no selectivos de la ciclooxigenasa, enzima encargada de la síntesis de prostaglandinas, los cuales tienen diferentes efectos hemodinámicos. Por un lado, se postula que de las dos isoenzimas conocidas de la ciclooxigenasa (COX-1 y COX-2), la COX-1 sería la implicada en la fisiología basal renal y de ahí su efecto nocivo., ${ }^{4,9}$

De los 16 pacientes que presentaron las complicaciones, el 18,7\% fueron antes de las 72 horas de iniciar el diclofenaco, en $12,6 \%$ durante la aplicación del ciclo y en $68,7 \%$ fueron 72 horas después. En nuestro estudio se encontró que en la mayor parte de los casos después del cierre de DAP se logró pronto destete del ventilador y el requerimiento de oxígeno suplementario a 28 días fue de $61,3 \%$. No se valoró la evolución neurológica a largo plazo, lo que sería interesante para próximas revisiones, ya que se ha observado también que el uso de estos medicamentos resulta en disminución de la circulación y la oxigenación cerebral, con implicaciones sobre el neurodesarrollo. ${ }^{9,16}$

No se puede relacionar la permanencia hospitalaria con el cierre del DAP, ya que esta puede ser influenciada por otros factores no valorados en el estudio. La mortalidad reportada fue de ocho casos por todas las causas, que se presentaron en $75 \%$ después de las 72 horas tras la administración del medicamento. El grupo corresponde a pretérminos extremos de muy bajo peso al nacer, con antecedentes maternos de sepsis y embarazos no controlados, quienes cursaron con sepsis neonatal, falla orgánica múltiple y patologías propias del prematuro con grados variables de severidad como hemorragia intraventricular, enfermedad de membrana hialina y falla renal.

Dentro de los hallazgos que puedan sugerir la presencia de DAP, en la cohorte del estudio se observó que la sospecha clínica dada por hiperdinamia y soplo cardíaco fueron los determinantes para realizar ecocardiograma. Consideramos que deben estar dentro del protocolo para todos los $\mathrm{RN}$ pretérmino con el fin de brindar una adecuada atención.

Conclusiones e implicaciones para la práctica: el diclofenaco presenta algunos parámetros que lo hacen competente para el cierre del DAP, con mayor disponibilidad en el mercado y facilidad de adquisición que las actuales opciones terapéuticas (indometacina, ibuprofeno $)^{7}$, lo cual hace atractivo su uso en la práctica clínica. En cuanto a la seguridad, nuestros datos reflejan un adecuado margen de confianza para su uso, se produjo un bajo porcentaje de efectos adversos relacionados con el grupo farmacológico del medicamento ${ }^{7}$ si se compara con la terapia estándar. Con base en la información disponible, el diclofenaco no proporciona beneficios netos sobre las alternativas para el tratamiento del DAP. Los AINES se pueden 
utilizar pero el médico especialista debe saber que estos se asocian con efectos que pueden ser graves. ${ }^{16}$ Las investigaciones futuras se deben centrar en el seguimiento a largo plazo (complicaciones y beneficios tardíos) hasta al menos 24 meses de edad corregida y la comparación con otras opciones terapéuticas, teniendo en cuenta la posibilidad de realizar estudios multicéntricos.

\section{Referencias}

1. Gimeno NA, Cano SA, Fernández GC, Carrasco Moreno JI, Izquierdo MI, Gutiérrez LA, et al. [Ibuprofen versus indomethacin in the treatment of patent ductus arteriosus in preterm infants]. $2005 \mathrm{Sep}$;(3):212-8.

2. Evans N. Current controversies in the diagnosis and treatment of patent ductus arteriosus in preterm infants. 2003 Aug;(4):168-77.

3. Vanhaesebrouck S, Zonnenberg I, Vandervoort P, Bruneel E, Van Hoestenberghe MR, Theyskens C. Conservative treatment for patent ductus arteriosus in the preterm. $2007 \mathrm{Jul}$;(4):F244-F247.

4. Weir EK, López-Bameo J, Buckler KJ, Archer SL. Acute oxygen-sensing mechanisms. 2005 Nov 10;(19):2042-55.

5. Cooke L, Steer P, Woodgate P. Indomethacin for asymptomatic patent ductus arteriosus in preterm infants. 2003;(2):CD003745.

6. Golombek SG, Sola A, Baquero H, Borbonet D, Cabanas F, Fajardo C, et al. [First SIBEN clinical consensus: diagnostic and therapeutic approach to patent ductus arteriosus in premature newborns]. 2008 Nov;(5):454-81.

7. van OB, Smets K, Lecoutere D, Van de Broek H, Weyler J, Degroote K, et al. A comparison of ibuprofen and indomethacin for closure of patent ductus arteriosus. 2000 Sep 7;(10):674-81.

8. Eichenwald EC, Stark AR. Management and outcomes of very low birth weight. 2008 Apr 17;(16):1700-11.

9. Hamrick SE, Hansmann G. Patent ductus arteriosus of the preterm infant. 2010 May;(5):1020-30.
10. Shah SS, Ohlsson A. Ibuprofen for the prevention of patent ductus arteriosus in preterm and/or low birth weight infants. 2006;(1):CD004213.

11. Su BH, Lin HC, Chiu HY, Hsieh HY, Chen HH, Tsai YC. Comparison of ibuprofen and indometacin for early-targeted treatment of patent ductus arteriosus in extremely premature infants: a randomised controlled trial. 2008 Mar;(2):F94-F99.

12. GorkAS, Ehrenkranz RA, Bracken MB. Continuousinfisionversus intermittent bolus doses of indomethacin for patent ductus arteriosus closure in symptomati preterm infant. 2008;(1):CD006071.

13. Malviya M, Ohlsson A, Shah S. Surgical versus medical treatment with cyclooxygenase inhibitors for symptomatic patent ductus arteriosus in preterm infants. 2008;(1):CD003951.

14. Ohlsson A, Walia R, Shah S. Ibuprofen for the treatment of patent ductus arteriosus in preterm and/or low birth weight infants. 2008;(1):CD003481.

15. DiMenna L, Laabs C, McCoskey L, Seals A. Management of the neonate with patent ductus arteriosus. 2006 Oct;(4):333-40.

16. Schmidt B, Davis P, Moddemann D, Ohlsson A, Roberts RS, Saigal S, et al. Long-term effects of indomethacin prophylaxis in extremely-lowbirth-weight infants. 2001 Jun 28:(26): 1966-72.

17. Alano MA, Ngougmna E, Ostrea EM, Jr., Konduri GG. Analysis of nonsteroidal antiinflammatory drugs in meconium and its relation to persistent pulmonary hypertension of the newborn. 2001 Mar;(3):519-23.

18. Auer M, Brezinka C, Eller P, Luze K, Schweigmann U, Schwarzler P. Prenatal diagnosis of intrauterine premature closure of the ductus arteriosus following maternal diclofenac application. 2004 May;(5):513-6.

19. Luchese S, Manica JL, Zielinsky P. Intrauterine ductus arteriosus constriction: analysis of a historic cohort of 20 cases. 2003 Oct;(4):405-4

20. Paul AD, Chauhan CK. Study of usage pattern of nonsteroidal antiinflammatory drugs (NSAIDs) among different practice categories in Indian clinical setting. $2005 \mathrm{Feb} ;(12): 889-92$.

21. Siu KL, Lee WH. Maternal diclofenac sodium ingestion and severe neonatal pulmonary hypertension. 2004 Mar;(3):152-3.

22. Torloni MR, Cordioli E, Zamith MM, Hisaba WJ, Nardozza LM, Santana $\mathrm{RM}$, et al. Reversible constriction of the fetal ductus arteriosus after maternal use of topical diclofenac and methyl salicylate. $2006 \mathrm{Feb}$;(2):227-9. 